OPEN ACCESS

Edited by:

Hsing-Chun Kuo,

Kaohsiung Chang Gung Memorial

Hospital, Taiwan

Reviewed by:

Ying-Hsien Huang,

Kaohsiung Chang Gung Memorial

Hospital, Taiwan

Hsing-Chun Kuo,

Chang Gung University of Science

and Technology, Taiwan

*Correspondence:

Chunyi Liu

chunyi618@163.com

Specialty section:

This article was submitted to

Pediatric Immunology,

a section of the journal

Frontiers in Pediatrics

Received: 30 June 2019 Accepted: 04 October 2019 Published: 01 November 2019

Citation:

Jin P, Luo Y, LiU X, Xu J and LiU C (2019) Kawasaki Disease Complicated With Macrophage Activation Syndrome: Case Reports and

Literature Review.

Front. Pediatr. 7:423.

doi: 10.3389/fped.2019.00423

\section{Kawasaki Disease Complicated With Macrophage Activation Syndrome: Case Reports and Literature Review}

\author{
Ping Jin ${ }^{1}$, Yong Luo ${ }^{1}, X i$ Liu $^{2}$, Jinji Xu ${ }^{1}$ and Chunyi Liu ${ }^{1 *}$ \\ ${ }^{1}$ Pediatric Intensive Care Unit, Baoan Maternal and Child Health Hospital, College of Medicine, Jinan University, Shenzhen, \\ China, ${ }^{2}$ Department of Cardiology, Baoan Maternal and Child Health Hospital, College of Medicine, Jinan University, \\ Shenzhen, China
}

Macrophage activation syndrome (MAS) is a rare and severe complication of Kawasaki disease (KD). The clinical feature, early diagnosis and treatment options, and prognosis need to be further determined in patients with KD complicated with MAS. In this report, we retrospectively analyzed three KD patients complicated with MAS who were treated in pediatric intensive care units (PICU) and reviewed the relevant literatures. We find that being male, being age over 2 years old, incomplete $\mathrm{KD}$, intravenous immunoglobulin (IVIG) non-responder, or persistent fever greater than 10 days are all highly associated with occurrence of MAS. Additional work-ups should be performed promptly in patient with above predisposing factors to rule out complication of MAS. Patients with KD complicated with MAS are at a higher risk of having coronary artery involvement or aneurysm formation, which can be reversed with timely treatment. Early identification and prompt treatment are key points for improving the prognosis of KD patients complicated with MAS.

\section{Keywords: Kawasaki disease, complication, macrophage activation syndrome, coronary artery, children}

\section{INTRODUCTION}

Kawasaki disease (KD), also known as mucocutaneous lymph node syndrome, is one of the most common forms of vasculitis in children (1). When untreated, manifestations of acute inflammation and fever can last on average of 12 days (2), and may also manifest in a variety of complications including coronary artery aneurysm which has a reported incidence rate of up to $25 \%$ in untreated $\mathrm{KD}$ patients $(3,4)$. However, the incidence of aneurysm formation decreases to $3-5 \%$ in $\mathrm{KD}$ cases following IVIG treatment. The other non-cardiac complications include shock, renal diseases, gastrointestinal involvement, macrophage activation syndrome (MAS), sensory nerve deafness (5-8). MAS is a rare and severe complication of KD with an incidence of $1.1-1.9 \%$ of all KD patients $(9,10)$.

MAS is a type of hemophagocytic lymphohistiocytosis (HLH) that occurs under the circumstance of rheumatic diseases and is a life-threatening excessive immune activation syndrome. It is commonly seen in systemic-onset juvenile idiopathic arthritis (sJIA), with incidence rates as high as $8-17 \%$ and is also reported in pediatric patients with KD. There were only 8 cases of KD patients complicated with MAS reported between 1979 and 2007 on MEDLINE (11). As of September 2016, the case report of KD complicated with MAS have increased to 69 cases, but only 34 of them had data to confirm the diagnosis of MAS (12). Therefore, MAS is still a rare entity 
compared to other complications in patients with $\mathrm{KD}$, with an occurrence rate less than 2\% (7). Of interesting note, the incidence rate of coronary artery abnormalities in $\mathrm{KD}$ complicated with MAS is as high as $46 \%$, which is much higher than that of KD patients which resolve on its own (15-25\%) (12).

This report retrospectively analyzes three $\mathrm{KD}$ patients complicated with MAS who were treated in the pediatric intensive care unit (PICU) of our hospital and reviews the relevant literature in order to further our understanding of this disease and make an earlier diagnosis of this complication to improve prognosis through timely treatment.

\section{CASE REPORT}

\section{Case 1}

A 4 year and 8-month-old girl initially presents to us with 6 days of fever with $\operatorname{Tmax} 40^{\circ} \mathrm{C}$ and 2 days of dysmorphic skin rash. She has also been having chills, intermittent abdominal discomfort, and occasional cough during this period of time. Physical exam notable for cervical lymphadenopathy (between 1.5 and $2.0 \mathrm{~cm}$ ), strawberry tongue and fissure lips, diffuse maculopapular rash. The diagnosis of incomplete $\mathrm{KD}$ was suspected. On the third day of admission, a cardiac ultrasound showed coronary artery dilatation with the left main coronary artery (LMCA) $3.0 \mathrm{~mm}$ (Z-score +2.50 ), and the right coronary artery (RCA) $2.69 \mathrm{~mm}$ (Z-score +2.39) (Z-scores were calculated via http://www.tspc.org.tw:80/service/z_score.asp) in diameter. The influenza virus FLUL-A antigen was positive $(+)$. The patient was treated with aspirin (30 mg/kg/day) (13) and a single dose of intravenous immunoglobulin (IVIG) at $2 \mathrm{~g} / \mathrm{kg}$ infusion within $12 \mathrm{~h}$ after above finding on cardiac ultrasound. Fever persisted $36 \mathrm{~h}$ after completing IVIG treatment. The patient was deemed IVIG-nonresponsive after a second dose of IVIG $(2 \mathrm{~g} / \mathrm{kg})$ was administrated and fever, skin rash, and hepatosplenomegaly continued to be noted with little or no improvement. On the sixth day of admission, laboratory data showed platelets at $85 \times$ $10^{9} / \mathrm{L}$, ferritin at $10,615 \mathrm{ng} / \mathrm{ml}$, and triglycerides at $3.42 \mathrm{mmol} / \mathrm{L}$. Hemophagocytic phenomenon was not detected by cytology from bone marrow aspiration, but hemophagocytosis is indicated in her cervical lymph node biopsy. Therefore the patient was diagnosed KD complicated with MAS (HLH-2004) and was initiated on methylprednisolone pulse treatment at $30 \mathrm{mg} / \mathrm{kg} . \mathrm{d}$ for 3 days, followed with chemotherapy (VP-16) according to the HLH-2004 protocol, with a total treatment course of 11 weeks. At the 3-month clinic follow-up visit, the repeat cardiac ultrasound showed normal origin of LMCA at $2.3 \mathrm{~mm}(\mathrm{Z}$ score +1.05$)$ and RCA at $1.6 \mathrm{~mm}(\mathrm{Z}$-score +0.14$)$ in diameter.

\section{Case 2}

This is a 2 year and 6-month-old boy, who presents to us for a cough that has lasted more than half a month and a recurrent fever 4 days. Eye conjunctival congestion, dry and chapped lips, strawberry tongue, and rash were noted on admission physical exam. On the third day of admission, a cardiac ultrasound showed enlarge LMCA at $3.58 \mathrm{~mm}(\mathrm{Z}$-score +3.66) and RCA at $2.1 \mathrm{~mm}$ (Z-score +1.47$)$. The boy was then diagnosed with incomplete $\mathrm{KD}$ and was infused with IVIG (2 g/kg/day). $36 \mathrm{~h}$ after completing IVIG treatment, the patient exhibited persistent fever, poor spirit, and dyspnea and was then transferred to the PICU. Blood gas showed that the P/F ratio (ratio between the $\mathrm{PaO}_{2}$ and the inspired oxygen concentration expressed as a fraction) was $<200$. The patient underwent endotracheal intubation and mechanical ventilation. Bronchoalveolar lavage fluid was positive for adenovirus and Mycoplasma pneumoniae. Fever and hepatosplenomegaly continued to be noted 3 days after transfer to PICU. Laboratory data revealed hemoglobin $79 \mathrm{~g} / \mathrm{L}$, platelets $95 \times 10^{9} / \mathrm{L}$, serum ferritin $3,594 \mathrm{ng} / \mathrm{ml}$, and triglycerides $3.14 \mathrm{mmol} / \mathrm{L}$. Bone marrow cytology did not detect the hemophagocytic phenomenon. The patient was considered to be IVIG-nonresponsive KD complicated with MAS (Table 1), and a second dose of IVIG was administered after plasma exchange. The patient's clinical symptoms were alleviated. Cardiac ultrasound at the 3-month follow-up showed LMCA at $2.50 \mathrm{~mm}(\mathrm{Z}$-score +1.72$)$ and RCA at $1.92 \mathrm{~mm}(\mathrm{Z}$-score +1.08$)$.

\section{Case 3}

This case is a 5 year and 1-month old boy who presented with a history of fever for 5 days and sudden unconsciousness for $2 \mathrm{~min}$ (spontaneously recovered afterwards). His highest temperature was $40^{\circ} \mathrm{C}$ and was associated with chills and occasional cough. Physical examination prominent for red, dry, and chapped lips, strawberry tongue, and enlarged cervical lymph node $(1.5 \mathrm{~cm})$. An electroencephalography (EEG) was abnormal (large number of spike-waves/slow spike-waves activated in the right central region, frontal area, right center, and central midline during admission). Given continued fever, atypical KD was suspected, and a cardiac ultrasound was performed on the fourth day of admission and showed LMCA at $2.96 \mathrm{~mm}(\mathrm{Z}$-score +2.23$)$ and $\mathrm{RCA}$ at $2.90 \mathrm{~mm}(\mathrm{Z}$-score +2.57$)$. The patient was diagnosed with incomplete $\mathrm{KD}$ and was administered IVIG (2 g/kg/d). As fever persisted $36 \mathrm{~h}$ after the IVIG treatment, further work up with lab studies showed white blood cell count $2.94 \times$ $10^{9} / \mathrm{L}$, hemoglobin $88 \mathrm{~g} / \mathrm{L}$, platelets $78 \times 10^{9} / \mathrm{L}$, triglycerides 2.31 $\mathrm{mmol} / \mathrm{L}$, and serum ferritin $712.60 \mathrm{ng} / \mathrm{ml}$. Bone marrow cytology did not detect the hemophagocytic phenomenon. The patient was diagnosed with incomplete KD complicated with MAS (Table 1, according to sJIA-MAS 2016). The patient then received a second dose of IVIG $(2 \mathrm{~g} / \mathrm{kg} / \mathrm{d})$ and 3 days of IV methylprednisolone (30 mg/kg/d), followed with improvement of symptoms and resolution of fever. His cardiac ultrasound at the 3-month followup visit showed LMCA at $2.26 \mathrm{~mm}(\mathrm{Z}$-score +0.77$)$ and RCA at $2.02 \mathrm{~mm}$ (Z-score +1.01$)$.

\section{DISCUSSION}

$\mathrm{KD}$ is a common vasculitis in children, and its incidence is highest among children living in East Asia or Asian children living in other regions of the world (14). Clinical manifestations of KD primarily include systemic inflammation and skin mucosal inflammation. The diagnosis and treatment guidelines by the American Heart Association in 2017 proposes a diagnosis of $\mathrm{KD}$ based on more than 5 days of fever and $\geq 4$ main clinical characteristics. If a child has fever for $\geq 5$ days and meets 2 or 3 main clinical characteristics combined with coronary artery 


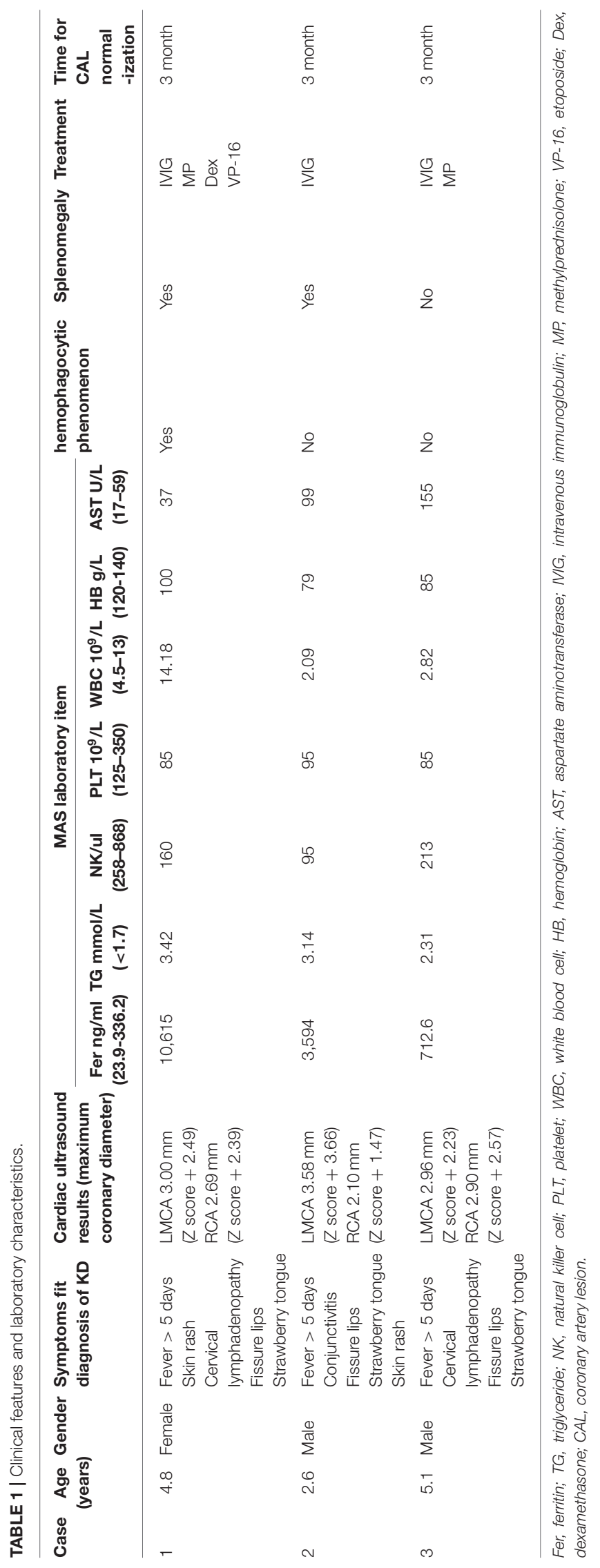

dilatation, the child can also be diagnosed with KD (incomplete $\mathrm{KD}$ ), except for exudative conjunctivitis, exudative pharyngitis, ulcerative stomatitis, bullous or vesicular rash, and systemic lymphadenopathy or splenomegaly $(3,15)$.

The three cases in our report all met the clinical diagnostic criteria for incomplete KD. Cardiac ultrasound of all three showed coronary artery dilatation, among which two cases met the criteria for a small coronary artery aneurysm (3). All three patients have persistent fever at $36-48 \mathrm{~h}$ after administration of a single dose of IVIG at $2 \mathrm{~g} / \mathrm{kg}$, indicating nonresponsiveness to IVIG treatment. In this study, 10 days after onset, the disease progressed rapidly and manifested as persistent fever, hepatosplenomegaly, pancytopenia, significant elevation of ferritin and hypertriglyceridemia. These symptoms are well consistent with clinical features and laboratory characteristics of MAS. Thus, the three cases presented here met the diagnosis of IVIG-nonresponsive KD complicated with MAS.

The incidence rate of KD complicated with MAS is 1.1-1.9\% $(9,10)$. The risk of KD complicated with MAS increases 7 -fold in KD patients over the age of 5 years old (16). Eighty-five percent of KD occurs in children under the age of 2 years old. In this report, all three patients were over 2 years old and older than the common age of KD onset. Kato et al. found that $49.3 \%$ of patients with KD complicated with MAS were over 5 years old (17).

MAS may occur in any stage of KD (acute stage, subacute stage, or recovery stage) and may also occur prior to a $\mathrm{KD}$ diagnosis, but in most cases, it appears simultaneously with $\mathrm{KD}$ (12). The MAS in the three cases of this study also presented simultaneously with $\mathrm{KD}$. The mortality rate of MAS is $8-22 \%(18,19)$, and prompt treatment is the key positive factor for survival of patients with MAS. Therefore, earlier diagnosis of MAS in children with $\mathrm{KD}$ is of most importance for prompt treatments and better prognosis. The early diagnosis of KD complicated with MAS is a clinical challenge as MAS lacks specific diagnostic features and has many overlapping manifestations and laboratory findings with $\mathrm{KD}$. For example, persistent fever, skin rash, thrombocytopenia, abnormal liver enzymes, anemia (20), and hypertriglyceridemia can be found both in KD and MAS. However, previous reports showed KD patients with ICU admission tended to have significant anemia and thrombocytopenia $(21,22)$. Thrombocytopenia was common in the acute critical stage, whereas thrombocytosis was thought to be a feature of later phase. Early thrombocytopenia was considered a risk marker for coronary aneurysm formation (2). Despite the overlap symptoms between KD and MAS, a serial dynamic echocardiogram test was crucial for differentiation and diagnosis especially when the two diseases occurred together (23). Our study suggests that clinical high vigilance and frequent surveillance of MAS is very helpful for diagnosis of this entity, especially in setting of fever persisting more than 10 days or IVIG-nonresponsive KD patients.

The diagnosis criteria for rheumatic diseases complicated with MAS are mainly provided by the HLH-2004 diagnostic criteria established by the International HLH Association (24). Furthermore, the HLH-2004 criteria cannot be applied for the clinical application of secondary HLH, including sJIA complicated with MAS (25). The European League Against 
Rheumatism (EULAR) together with the American College of Rheumatology (ACR) published the diagnostic criteria for sJIA complicated with MAS as sJIA-MAS (26), which also suggested an application for KD complicated with MAS (27). For febrile patients with confirmed or suspected sJIA and ferritin > $684 \mathrm{ng} / \mathrm{ml}$, the sJIA-MAS 2016 suggests that they can be diagnosed with sJIA-MAS as long as they meet any 2 or more of the following criteria: platelet count $\leq 181$ $\times 10^{9} / \mathrm{L}$, aspartate aminotransferase $>48 \mathrm{U} / \mathrm{L}$, triglycerides $>1,560 \mathrm{mg} / \mathrm{L}$, and fibrinogen $\leq 3,600 \mathrm{mg} / \mathrm{L}$. In this study, cases 1 and 2 met the diagnostic criteria for HLH-2004, and case 3 met the diagnostic criteria for sJIA-MAS 2016. However, the sJIA-MAS 2016 diagnostic criteria were published recently, and more clinical applications are still needed before a final determination.

Approximately one-third of HLH patients have nervous system abnormalities, including seizures, altered mental status, and ataxia (28). Those neurological manifestations can either be the main reason for seeking medical care or sometimes as an initial clinical presentation (29). Magnetic resonance imaging (MRI) of the brains of HLH patients may show low-density or necrotic areas (30). Cerebrospinal fluid abnormality could be seen in $50 \%$ of the patients, and their risk of neurological sequelae increases (31). Altered consciousness and abnormal EEG occurring prior to KD symptoms such as in case 3 of our study, is similar to previous reports (29).

Although hemophagocytic phenomenon can be used as a marker for the overactivation of macrophages, it is not a specific manifestation of HLH and does not always occur in the early stage of MAS. Furthermore, bone marrow aspiration is an invasive procedure that is not included in the sJIAMAS 2016 (26). Hemophagocytic phenomenon could not be found in any of the MAS cases (25-100\%) from bone marrow examination (32). Some patients may experience the hemophagocytic phenomenon in the late stage of the disease after clinical symptoms are alleviated (23). In our patients, bone marrow cytology examinations showed a negative finding of hemophagocytic phenomenon in all three cases, but they fit the diagnosis of MAS. MAS can be diagnosed if patients meet clinical and laboratory characteristics, even in the absence of the hemophagocytic phenomenon in the bone marrow.

Splenomegaly is rarely present in patients with $\mathrm{KD}$ but occurs in $69 \%$ of patients with KD complicated with MAS (12). KD patients should be considered for the possibility of MAS if persistent fever and presence of splenomegaly are noted (12). Thrombocytopenia is a high risk factor for acute myocardial infarction or coronary aneurysm in $\mathrm{KD}$ patients (33). In our patients, all three had thrombocytopenia and coronary artery dilatation, of which two had small coronary aneurysms. The coronary arteries of all three patients regressed

\section{REFERENCES}

1. Burns JC, Glode MP. Kawasaki syndrome. Lancet. (2004) 364 533-44. doi: 10.1016/S0140-6736(04)16814-1 to normal range within 3 months after disease onset. The findings of this report indicate a high incidence rate (100\%) of coronary abnormality in patients with $\mathrm{KD}$ complicated with MAS and a high regression rate following appropriate treatment were observed.

A summary of this case report of three $\mathrm{KD}$ patients complicated with MAS shows its following clinical features: (1) more common in male gender patients, that is consistent with the report of literature (34); (2) more likely in patients older than 2 years old than patient with typical KD who are less than 2 years old; (3) fever persistent more than 10 days; (4) splenomegaly; (5) non-responsiveness to IVIG; and (6) incomplete KD.

MAS is a rare complication of $\mathrm{KD}$ with serious clinical implications. It commonly occurs with coronary involvement. Additional treatment should be given to patients who are not completely responsive to the initial IVIG treatment, including a second course of IVIG, glucocorticoid, tumor necrosis factor (TNF) inhibitor, immunosuppressant agents, or plasma exchange. Both HLH-2004 or sJIA-MAS 2016 has provided diagnosis criteria for KD with MAS, even when bone marrow aspiration has negative findings.

\section{DATA AVAILABILITY STATEMENT}

All datasets generated for this study are included in the manuscript/supplementary files.

\section{ETHICS STATEMENT}

The studies involving human participants were reviewed and approved by the Ethics Committee of Baoan Maternal and Child Health Hospital. Written informed consent to participate in this study was provided by the participants' legal guardian/next of kin. Written informed consent was obtained from the individual(s), and minor(s)' legal guardian/next of kin, for the publication of any potentially identifiable images or data included in this article.

\section{AUTHOR CONTRIBUTIONS}

PJ observed the patients, collected and analyzed the data, and drafted the manuscript. YL and JX helped to observe the patients. XL participated in writing the study. CL coordinated the execution of the studies and corrected the manuscript. All authors read and approved the final manuscript.

\section{FUNDING}

This study was supported by grants from the Sanming Project of Medicine in Shenzhen (No. SZSM201606088).

2. Newburger JW, Takahashi M, Gerber MA, Gewitz MH, Tani LY, Burns JC, et al., Diagnosis, treatment, and long-term management of Kawasaki disease: a statement for health professionals from the Committee on Rheumatic Fever, Endocarditis and Kawasaki Disease, Council on Cardiovascular Disease in 
the Young, American Heart Association. Circulation. (2004) 110:2747-71. doi: 10.1161/01.CIR.0000145143.19711.78

3. McCrindle BW, Rowley AH, Newburger JW, Burns JC, Bolger AF, Gewitz $\mathrm{M}$, et al. Diagnosis, treatment, and long-term management of Kawasaki disease: a scientific statement for health professionals from the American Heart Association. Circulation. (2017) 135:e927-99. doi: 10.1161/CIR.0000000000000484

4. Kuo HC, Yang KD, Chang WC, Ger LP, Hsieh KS. Kawasaki disease: an update on diagnosis and treatment. Pediatr Neonatol. (2012) 53:4-11. doi: 10.1016/j.pedneo.2011.11.003

5. Kanegaye JT, Wilder MS, Molkara D, Frazer JR, Pancheri J, Tremoulet AH, et al. Recognition of a Kawasaki disease shock syndrome. Pediatrics. (2009) 123:e783-9. doi: 10.1542/peds.2008-1871

6. Magalhaes CM, Magalhaes Alves NR, Oliveira KM, Silva IM, Gandolfi L, Pratesi R. Sensorineural hearing loss: an underdiagnosed complication of Kawasaki disease. J Clin Rheumatol. (2010) 16:322-5. doi: 10.1097/RHU.0b013e3181f603bc

7. Veiga PA, Pieroni D, Baier W, Feld LG, Association of Kawasaki disease and interstitial nephritis. Pediatr Nephrol. (1992) 6:421-3. doi: 10.1007/BF00873999

8. Zulian F, Falcini F, Zancan L, Martini G, Secchieri S, Luzzatto C, et al. Acute surgical abdomen as presenting manifestation of Kawasaki disease. J Pediatr. (2003) 142:731-5. doi: $10.1067 / \mathrm{mpd} .2003 .232$

9. Wang W, Gong F, Zhu W, Fu S, Zhang Q. Macrophage activation syndrome in Kawasaki disease: more common than we thought? Semin Arthritis Rheum. (2015) 44:405-10. doi: 10.1016/j.semarthrit.2014.07.007

10. al-Eid W, al-Jefri A, Bahabri S, al-Mayouf S. Hemophagocytosis complicating Kawasaki disease. Pediatr Hematol Oncol. (2000) 17:323-9. doi: $10.1080 / 088800100276316$

11. Tristano AG, Macrophage activation syndrome: a frequent but underdiagnosed complication associated with rheumatic diseases. Med Sci Monit. (2008) 14:Ra27-36.

12. Garcia-Pavon S, Yamazaki-Nakashimada MA, Baez M, Borjas-Aguilar KL, Murata C. Kawasaki disease complicated with macrophage activation syndrome: a systematic review. J Pediatr Hematol Oncol. (2017) 39:445-51. doi: 10.1097/MPH.0000000000000872

13. Kuo HC, Guo MM, Lo MH, Hsieh KS, Huang YH. Effectiveness of intravenous immunoglobulin alone and intravenous immunoglobulin combined with high-dose aspirin in the acute stage of Kawasaki disease: study protocol for a randomized controlled trial. BMC Pediatr. (2018) 18:200. doi: 10.1186/s12887-018-1180-1

14. Kao AS, Getis A, Brodine S, Burns JC. Spatial and temporal clustering of Kawasaki syndrome cases. Pediatr Infect Dis J. (2008) 27:981-5. doi: 10.1097/INF.0b013e31817acf4f

15. Kuo HC, Preventing coronary artery lesions in Kawasaki disease. Biomed J. (2017) 40:141-6. doi: 10.1016/j.bj.2017.04.002

16. Atteritano M, David A, Bagnato G, Beninati C, Frisina A, Iaria C, et al. Haemophagocytic syndrome in rheumatic patients. A systematic review. Eur Rev Med Pharmacol Sci. (2012) 16:1414-24.

17. Kato S, Yoshimura K, Tanabe Y, Kimata T, Noda Y, Kawasaki H, et al. A child with Epstein-Barr Virus-associated hemophagocytic lymphohistiocytosis complicated by coronary artery lesion mimicking Kawasaki disease. J Pediatr Hematol Oncol. (2013) 35:e317-9. doi: 10.1097/MPH.0b013e31828e5281

18. Stephan JL, Kone-Paut I, Galambrun C, Mouy R, Bader-Meunier B, Prieur AM. Reactive haemophagocytic syndrome in children with inflammatory disorders. A retrospective study of 24 patients. Rheumatology. (2001) 40:128592. doi: 10.1093/rheumatology/40.11.1285

19. Sawhney S, Woo P, Murray KJ. Macrophage activation syndrome: a potentially fatal complication of rheumatic disorders. Arch Dis Child. (2001) 85:421-6. doi: 10.1136/adc.85.5.421

20. Huang YH, Kuo HC. Anemia in Kawasaki disease: hepcidin as a potential biomarker. Int J Mol Sci. 18 (2017) 18:820. doi: 10.3390/ijms18040820

21. Kuo CC, Lee YS, Lin MR, Hsia SH, Chen CJ, Chiu CH, et al. Characteristics of children with Kawasaki disease requiring intensive care: 10 years' experience at a tertiary pediatric hospital. J Microbiol Immunol Infect. (2018) 51:184-90. doi: 10.1016/j.jmii.2016.06.004

22. Chen PS, Chi H, Huang FY, Peng CC, Chen MR, Chiu NC. Clinical manifestations of Kawasaki disease shock syndrome: a case-control study. $J$ Microbiol Immunol Infect. (2015) 48:43-50. doi: 10.1016/j.jmii.2013.06.005

23. Jordan MB, Allen CE, Weitzman S, Filipovich AH, McClain KL. How I treat hemophagocytic lymphohistiocytosis. Blood. (2011) 118:4041-52. doi: 10.1182/blood-2011-03-278127

24. Henter JI, Horne A, Arico M, Egeler RM, Filipovich AH, Imashuku $\mathrm{S}$, et al. HLH-2004: diagnostic and therapeutic guidelines for hemophagocytic lymphohistiocytosis. Pediatr Blood Cancer. (2007) 48:124-31. doi: $10.1002 / p b c .21039$

25. Ravelli A, Magni-Manzoni S, Pistorio A, Besana C, Foti T, Ruperto N, et al. Preliminary diagnostic guidelines for macrophage activation syndrome complicating systemic juvenile idiopathic arthritis. J Pediatr. (2005) 146:598604. doi: 10.1016/j.jpeds.2004.12.016

26. Ravelli A, Minoia F, Davi S, Horne A, Bovis F, Pistorio A, et al. 2016 Classification criteria for macrophage activation syndrome complicating systemic juvenile idiopathic arthritis: A European League Against Rheumatism/American College of Rheumatology/Paediatric Rheumatology International Trials Organisation Collaborative Initiative. Ann Rheum Dis. (2016) 75:481-9. doi: 10.1002/art.39332

27. Han SB, Lee SY, Jeong DC, Kang JH. Should 2016 criteria for macrophage activation syndrome be applied in children with Kawasaki disease, as well as with systemic-onset juvenile idiopathic arthritis? Ann Rheum Dis. (2016) 75:e44. doi: 10.1136/annrheumdis-2016-209395

28. Jovanovic A, Kuzmanovic M, Kravljanac R, Micic D, Jovic M, Gazikalovic $\mathrm{S}$, et al. Central nervous system involvement in hemophagocytic lymphohistiocytosis: a single-center experience. Pediatr Neurol. (2014) 50:233-7. doi: 10.1016/j.pediatrneurol.2013.10.014

29. Deiva K, Mahlaoui N, Beaudonnet F, de Saint Basile G, Caridade G, Moshous D, et al. CNS involvement at the onset of primary hemophagocytic lymphohistiocytosis. Neurology. (2012) 78:1150-6. doi: 10.1212/WNL.0b013e31824f800a

30. Henter JI, Nennesmo I. Neuropathologic findings and neurologic symptoms in twenty-three children with hemophagocytic lymphohistiocytosis. J Pediatr. (1997) 130:358-65. doi: 10.1016/S0022-3476(97)70196-3

31. Horne A, Trottestam H, Arico M, Egeler RM, Filipovich AH, Gadner H, et al. Frequency and spectrum of central nervous system involvement in 193 children with haemophagocytic lymphohistiocytosis. Br J Haematol. (2008) 140:327-35. doi: 10.1111/j.1365-2141.2007.06922.x

32. Riviere S, Galicier L, Coppo P, Marzac C, Aumont C, Lambotte O, et al. Reactive hemophagocytic syndrome in adults: a retrospective analysis of 162 patients. Am J Med. (2014) 127:1118-25. doi: 10.1016/j.amjmed.2014. 04.034

33. Nofech-Mozes Y, Garty BZ. Thrombocytopenia in Kawasaki disease: a risk factor for the development of coronary artery aneurysms. Pediatr Hematol Oncol. (2003) 20:597-601. doi: 10.1080/08880010390 243004

34. Son MB, Gauvreau K, Ma L, Baker AL, Sundel RP, Fulton DR, et al. Treatment of Kawasaki disease: analysis of 27 US pediatric hospitals from 2001 to 2006. Pediatrics. (2009) 124:1-8. doi: 10.1542/peds.20 08-0730

Conflict of Interest: The authors declare that the research was conducted in the absence of any commercial or financial relationships that could be construed as a potential conflict of interest.

Copyright $\odot 2019 \mathrm{Jin}, \mathrm{Luo}, \mathrm{Liu}, \mathrm{Xu}$ and Liu. This is an open-access article distributed under the terms of the Creative Commons Attribution License (CC BY). The use, distribution or reproduction in other forums is permitted, provided the original author(s) and the copyright owner(s) are credited and that the original publication in this journal is cited, in accordance with accepted academic practice. No use, distribution or reproduction is permitted which does not comply with these terms. 